\title{
ANDES

\section{Correlación entre casos de diarrea aguda y búsquedas en Google en Perú}

\section{Correlation between cases of acute diarrhea and Google searches in Peru}

\author{
Hugo Arroyo-Hernández
}

aficina General de Información y Sistemas, Instituto Nacional de Salud, Lima, Perú

\section{Sr. Editor,}

En Perú las enfermedades diarreicas agudas son una causa importante de morbilidad en la niñez y se encuentran bajo vigilancia epidemiológica ${ }^{1}$. Diversos estudios han encontrado una correlación temporal entre las notificaciones de enfermedades infecciosas con las búsquedas en internet realizada por la población, por lo general para conocer más sobre las causas y el tratamiento ${ }^{2}$; sin embargo, no hay mucha información publicada en relación con uno de los síntomas gastrointestinales más comunes como lo es la diarrea.

Con el objetivo de determinar si existe una correlación entre los casos notificados de enfermedad diarreica aguda y las búsquedas de palabras afines en Google en Perú, se recopilaron datos de la proporción (0 a $100 \%$ ) calculada para el total de casos de enfermedad diarreica aguda para los grupos etarios $<1$ año, 1 a 5 años y $>5$ años notificados por el Centro Nacional de Epidemiologia, Prevención y Control de Enfermedades del Ministerio de Salud del Perú desde el año 2006 al 2020. Asimismo, se ingresó a la plataforma de Google Trends (www.google.com/trends) y se recopilaron los promedios de búsquedas que utilizaron las palabras "diarrea" y "rotavirus" por años. Este promedio refleja el interés de búsqueda para una región y periodo de- terminado donde un valor de 100 indica la popularidad máxima, 50 es la mitad de popular en relación con el valor máximo y 0 que no existen suficientes datos. Previa evaluación de la normalidad de las variables, se realizaron cálculos de correlación de Pearson (r2) considerando un valor $\mathrm{p}<0,05$ como estadísticamente significativo.

Se encontró un incremento de las búsquedas en Google para la palabra diarrea con una muy alta correlación con los casos notificados en mayores de 5 años ( $\mathrm{r} 2=0,91, \mathrm{p}<0,001)$. El incremento en la proporción de casos de enfermedad diarreica aguda en mayores de 5 años se explicaría por la disminución de los casos en menores de 5 años quienes representaban la mayoría de las notificaciones a mediados de la década del 2000, $\mathrm{y}$ en parte por las mejoras en acceso a servicios de agua y desagüe en la población ${ }^{3}$.

Se encontró además una alta correlación entre la proporción de casos notificados en menores de un año ( $\mathrm{r} 2=0,79, \mathrm{p}<0,001)$ y en niños de 1 a 5 años ( $\mathrm{r} 2=0,81$, $\mathrm{p}<0,001)$ con las búsquedas en Google de la palabra rotavirus (figura). La disminución de los casos de diarrea aguda en menores de cinco años podría tener relación con la introducción de la vacuna para rotavirus en el $2009^{1}$, un hallazgo similar fue encontrado en datos de hospitalización por gastroenteritis en los Estados

Correspondencia:

Hugo Arroyo-Hernández

hugoarroyo2007@gmail.com 


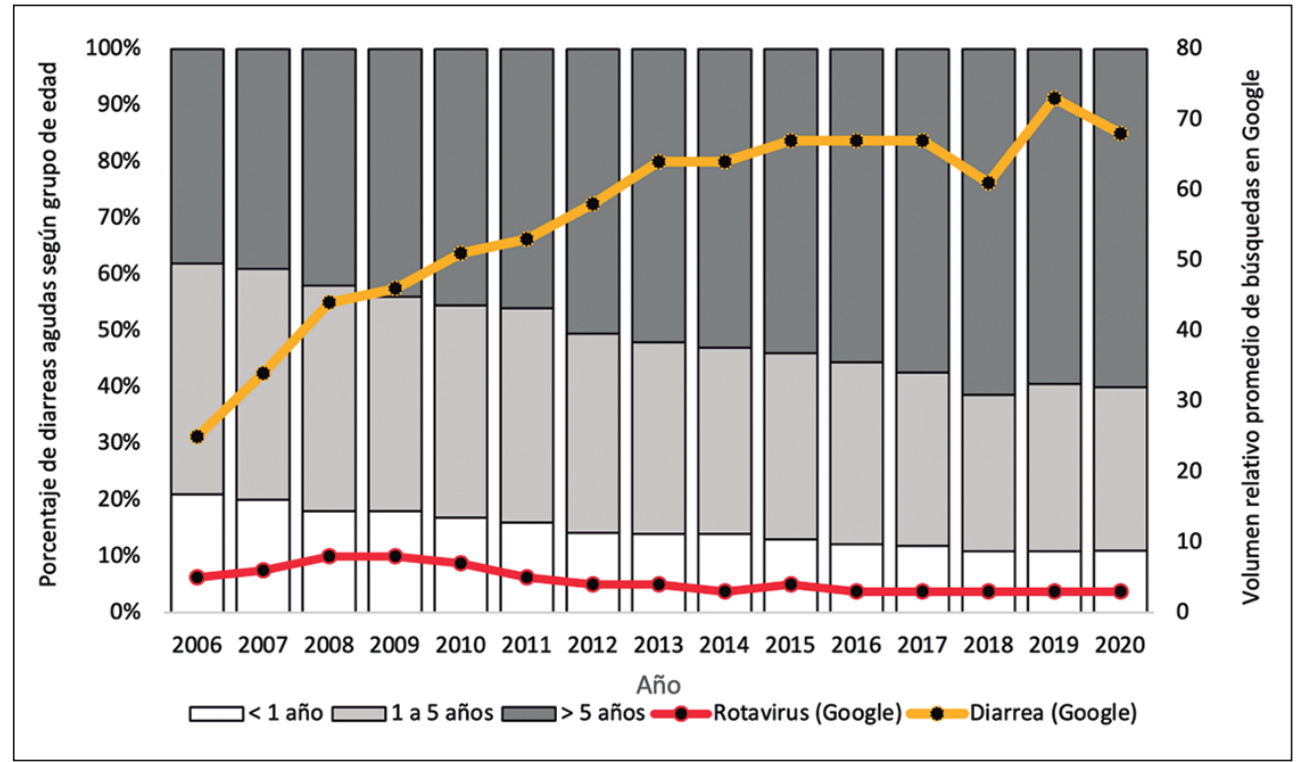

Figura. Distribución de episodios de enfermedad diarreica aguda según grupos de edad y búsquedas de las palabras rotavirus y diarrea en Google. Perú, 2006-2020.
Unidos y México ${ }^{4}$. Al ser el rotavirus una de las principales causas de diarrea en niños, la disminución en los casos podría tener un impacto en las búsquedas en Google sobre rotavirus realizadas por sus padres.

En conclusión, se encontró una correlación entre los casos de diarrea aguda notificados por el Ministerio de Salud del Perú y las búsquedas para las palabras diarrea y rotavirus realizadas en Google. Esto podría explicar que existe un impacto del comportamiento poblacional frente a una sintomatología gastrointestinal específica y el volumen de búsquedas realizadas en la internet. Al igual que otros estudios, se muestra que Google Trends ${ }^{5}$ podría ser una herramienta para comprender y rastrear los cambios en la epidemiología de algunos síntomas gastrointestinales de interés poblacional.

\section{Referencias}

1. Ordóñez IL. Situación epidemiológica de las Enfermedades Diarreicas Agudas (EDA) en el Perú 2019 a la SE 01- 2020. Boletín Epidemiológico del Perú. 2020; 29 (01):5-10.

2. Mavragani A, Ochoa G. Google Trends in Infodemiology and Infoveillance: Methodology Framework. JMIR Public
Health Surveill. 2019;5(2):e13439. http:// dx.doi: 10.2196/13439.

3. Arenas-Significación F, Gonzales-Medina C. Disminución de enfermedades infecciosas intestinales relacionada al acceso a servicios de agua y desagüe en el Perú, 2002-2009. An Fac Med. 2011; 72(4):245-8.

4. Shah MP, Lopman BA, Tate JE, et al. Use of Internet Search Data to Monitor
Rotavirus Vaccine Impact in the United States, United Kingdom, and Mexico. J Pediatric Infect Dis Soc. 2018;7(1):56-63. http://dx.doi: 10.1093/jpids/pix004.

5. Nuti SV, Wayda B, Ranasinghe I, et al. The use of google trends in health care research: a systematic review. PLoS One. 2014;9(10):e109583. http:// dx.doi: 10.1371/journal.pone.0109583. eCollection 2014 